\title{
Numerical Approach of the Influence of Geometric Properties on the Absorbing in Photonic Crystal
}

\author{
A. Merabti ${ }^{1, *}$, A. Hasni ${ }^{2, \dagger}$ M. Elmir ${ }^{2}$ \\ 1 Semiconductor Devices Physics Laboratory, TAHRI Mohamed University of Bechar, BP 417, Bechar, Algeria \\ 2 ENERGARID Laboratory, TAHRI Mohamed University of Bechar, BP 417, Bechar, Algeria
}

(Received 25 March 2016; published online 03 October 2016)

\begin{abstract}
In the proposed study, an investigation has been carried out in order to find a material efficient structure, capable of harnessing maximum solar spectrum. A material efficient structure designed using a one dimensional photonic crystal (1D PC) for amorphous silicon. Silicon material is used as it leads to environmental friendly design. The principal objective of this study is to maximize the photon absorption, keeping reflection to a minimum. The influence of geometric parameters on the absorption is studied by using the Finite element method (FEM). The results show that the absorption is affected by the geometry parameters. The optimum parameters of the proposed structure are period $(a=480 \mathrm{~nm})$, a filling factor $(f f=50 \%)$ and depth $(d=150 \mathrm{~nm})$. The increase of absorption in the lower region where the wavelengths are around $480 \mathrm{~nm}$, is explained by the reduction of the effective index resulting from the structure of the absorbent layer. For wavelengths between $480 \mathrm{~nm}$ and $600 \mathrm{~nm}$, the absorption is directly related to existing Fabry-Perot modes within the absorbent layer. Creating additional absorption peaks at wavelengths above about $600 \mathrm{~nm}$ weakly absorbed normally comes from the coupling of the incident light with slow Bloch modes of PC located above the light line.
\end{abstract}

Keywords: Dimensional structure, Finite element method (FEM), Absorption, Light trapping.

\section{INTRODUCTION}

Light trapping techniques are essential for increasing absorption of silicon solar cells. These techniques increases the efficiency of the solar cell. Understanding the absorption enhancement that is maximally achievable by light trapping schemes is thus of fundamental importance in solar cell research.

To increase the light harvesting efficiency, classical technologies combine the integration of an anti-reflection film (ARF), a textured top surface, and a reflector on the back side [1-4]. Traditional light trapping schemes used in photovoltaic cells are based on geometrical optics. Furthermore, the light trapping approaches based on wave optics can be capable of surpassing geometrical optics approaches in some cases [5].

In the past years, many wave optics light-trapping techniques have been explored such as plasmonics based designs [6,7], scattering into guided modes by metal nanoparticles [8], grating couplers [9], and photonic crystals (PCs) (in 1D [10], 2D [11] et 3D [12]).

Light interaction with such structures may yield phenomenon such as diffraction and optical band gaps which are caused by the wave nature of light Utilizing these phenomena may provide light trapping effects that are not achievable with conventional textures.

Photonic crystals are optical structures consist of dielectric materials with different refractive indexes. They have lattice constants and periodicity on the wavelength scale in one, two or three dimensions, and thus provide many interesting possibilities for "photon management" [13].

Have been studied extensively for several applications, including back reflector [14-15] or selective filters for tandem solar cells [16]

In this study, we proposed a one-dimensional photonic crystal (1D PC) for amorphous silicon. The influence of the geometrical parameters on the absorption is studied by using numerical calculations Finite element method (FEM). The results can be used to fabricate complete solar cells using such an absorbing structure.

\section{CALCULATION METHOD}

The Finite element method (FEM) is the method of choice for analysis, complex geometries and fast simulations of light interaction with photonic crystal [17].

The evolution of electric and magnetic fields in photonic crystal is described by Maxwell's equations. All information of light is contained in the electromagnetic fields, which is used to solve these differential equations. The Finite element method (FEM) performs rigorous simulations of Maxwell's equations.

This way of solving the Maxwell's equations allows the calculation of the reflectance $\mathrm{R}$, the transmittance $\mathrm{T}$, and thus of the absorption $A=1-R-T$ of a plane wave incident on our structures, as well as the plot of the electromagnetic field distribution in the structure.

Since the sunlight is generally unpolarized, the sensitivity of the proposed structure to the polarization state of the incident light has to be studied. Due to the modal properties of the 1D PC developed hereafter, most of the calculations will be done at normal incidence.

Finally, the integrated absorption of the structure is defined as the integration of the absorption taking into account the spectral irradiance of the sun (norm AM1.5). Thus, the integration is done from $350 \mathrm{~nm}$ to

\footnotetext{
*merabti73@yahoo.com

† hasni_haf@yahoo.fr
} 
$750 \mathrm{~nm}$ since the considered material does not absorb any more at larger wavelengths.

\section{PROPOSED STRUCTURE.}

Physical Model with Structural parameters are illustrated in Fig. 1, which consists of a periodic array of amorphous silicon a-Si strips.

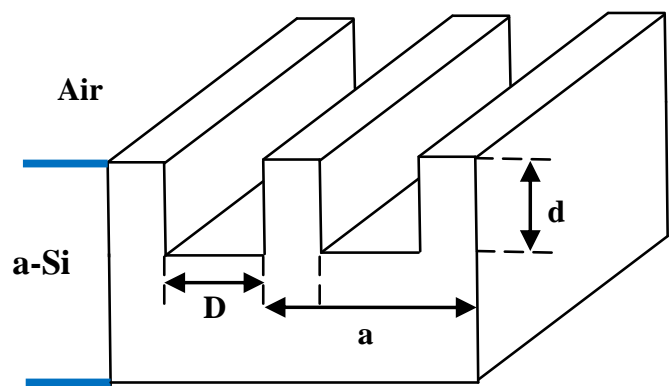

Fig. 1 - Physical Model of the Photonic Crystal

\section{RESULTS AND DISCUSSION}

\subsection{Influence of Depth $(d)$}

The influence of depth on absorption was calculated when the filling factor $(f f)$ and period were fixed at $50 \%$ and $480 \mathrm{~nm}$, respectively. The results are shown in Fig. 2a for TE and in Fig. 2b for TM wave. The absorption of unpatterned layer 1D PC a-Si is also shown for comparison.
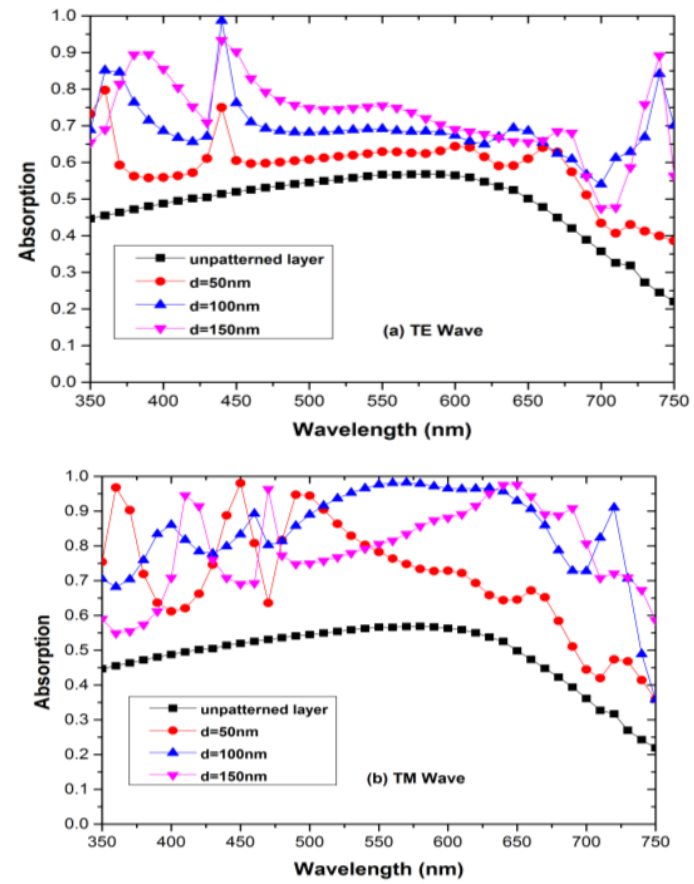

Fig. 2-Absorption of 1D PC a-Si for different depths, $f f=50 \%$ and $a=480 \mathrm{~nm}$ for (a) TE (b) TM wave

As shown in Fig. 2a and Fig. 2b, in the shorter wavelength region $(\lambda<550 \mathrm{~nm})$, we can find that the absorption of the $a$-Si increases strongly as the depth $(d)$ is increased. It is also observed that the absorption curve broadens as depth decrease.
Fig. 2a for TE shows that the absorption peak at wavelengths below $550 \mathrm{~nm}$ shifts towards the right with increase in depth. The absorption magnitude peak increases when the depth increases from 0 to $150 \mathrm{~nm}$.

Another peak in absorption begins to appear at around $675-750 \mathrm{~nm}$ range. The absorption is very sharp and close to unity for $d=100 \mathrm{~nm}$ at $\lambda=440 \mathrm{~nm}$.

It is evident from Fig. 3 that the high energy radiation is absorbed near the surface and fails to penetrate enough into the inside the amorphous silicon, at low wavelengths for TE and TM wave. And the simulated results for Max and Min Electric field are given in table1.
(1) 0.5

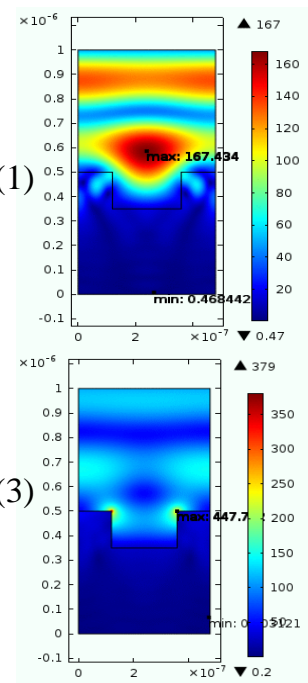

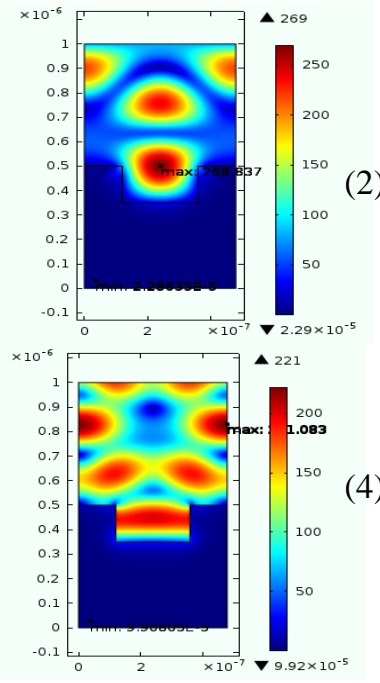

(2)
Fig. 3-Electric field intensities, calculated for the depth $(d=150 \mathrm{~nm})$ : (1-2) for TE wave, (3-4) for TM wave. The profiles in column I and II are corresponding to the wavelength of $400 \mathrm{~nm}$ and $550 \mathrm{~nm}$ respectively

\subsection{Influence of the Width $(D)$}

The influence of the width $(D)$ on the absorption is studied with different filling factor $(f f=D / a)$, where the depth and period are fixed at $150 \mathrm{~nm}$ and $480 \mathrm{~nm}$ for TM and TE respectively at normal incidence. The absorption of unpatterned layer 1D PC a-Si is also shown for comparison.

As shown in Fig.4a for TE wave, the Absorption spectra can be divided into two regions. In the first region (350 $\mathrm{nm} \leq \lambda \leq 560 \mathrm{~nm}$ ), the absorption spectra for the three structures $(f f=33.33 \%, f f=50.00 \%$ and $f f=66.67 \%$ ) are almost overlapping together. In the second region $(\lambda>560 \mathrm{~nm})$, the absorption peak begins to appear at around $560-650 \mathrm{~nm}$ range. It is also observed that the absorption curve broadens as the filling factor is increased.

The results in Fig. 4b show the filling factor does not affect the peak location but it influences the maximum value significantly.

At higher wavelengths, silicon has poor absorption, as a result of which the incident radiation penetrates deep into the substrate, as seen in Fig. 5. And the simulated results for Max and Min Electric field are given in Table 2. 
Table 1 - Max and Min Electric field calculated for the following depths (d)

\begin{tabular}{|c|c|c|c|c|c|}
\hline \multicolumn{2}{|c|}{} & \multicolumn{2}{|c|}{ TE wave } & \multicolumn{2}{c|}{ TM wave } \\
\hline \multirow{2}{*}{ depth (d) } & wavelength & $\begin{array}{c}\text { Max Electric } \\
\text { field (V/m) }\end{array}$ & $\begin{array}{c}\text { Min Electric } \\
\text { field (V/m) }\end{array}$ & $\begin{array}{c}\text { Max Electric } \\
\text { field (V/m) }\end{array}$ & $\begin{array}{c}\text { Min Electric } \\
\text { field (V/m) }\end{array}$ \\
\hline \multirow{3}{*}{$\begin{array}{c}\text { Unpatterned } \\
\text { layer }\end{array}$} & $\lambda=400 \mathrm{~nm}$ & 148.932 & $1.297 \mathrm{E}-6$ & 149.139 & $1.188 \mathrm{E}-5$ \\
\cline { 2 - 6 } & $\lambda=550 \mathrm{~nm}$ & 43.843 & 1.531 & 142.982 & 1.522 \\
\cline { 2 - 6 } & $\lambda=650 \mathrm{~nm}$ & 141.743 & 12.877 & 142.098 & 12.824 \\
\hline \multirow{3}{*}{$d=150 \mathrm{~nm}$} & $\lambda=400 \mathrm{~nm}$ & 268.837 & $2.286 \mathrm{E}-5$ & 221.283 & $9.908 \mathrm{E}-5$ \\
\cline { 2 - 6 } & $\lambda=550 \mathrm{~nm}$ & 167.434 & 0.468 & 447.772 & 0.203 \\
\cline { 2 - 6 } & $\lambda=650 \mathrm{~nm}$ & 151.286 & 0.168 & 361.424 & 1.429 \\
\hline
\end{tabular}
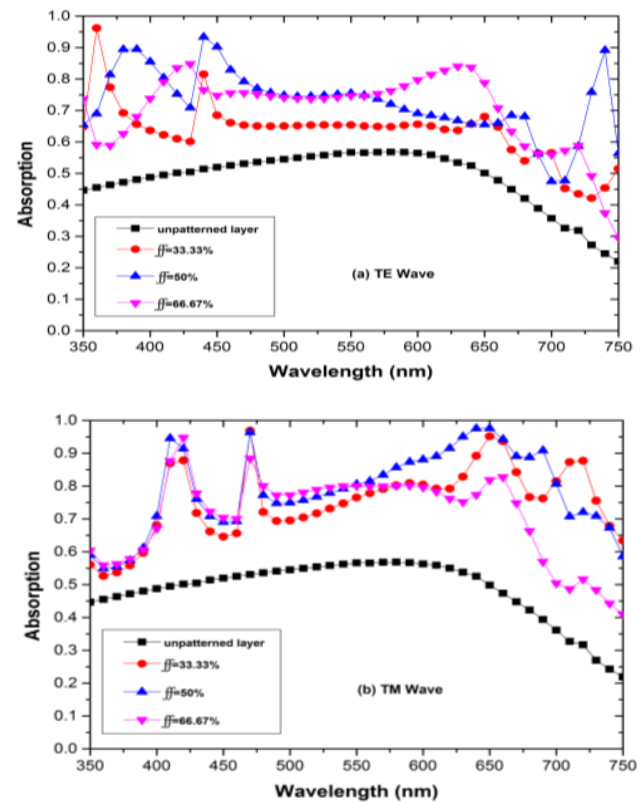

Fig. 4 - Absorption for different filling factor, $a=480 \mathrm{~nm}$ and $d=150 \mathrm{~nm}$ for (a) TE (b) TM wave
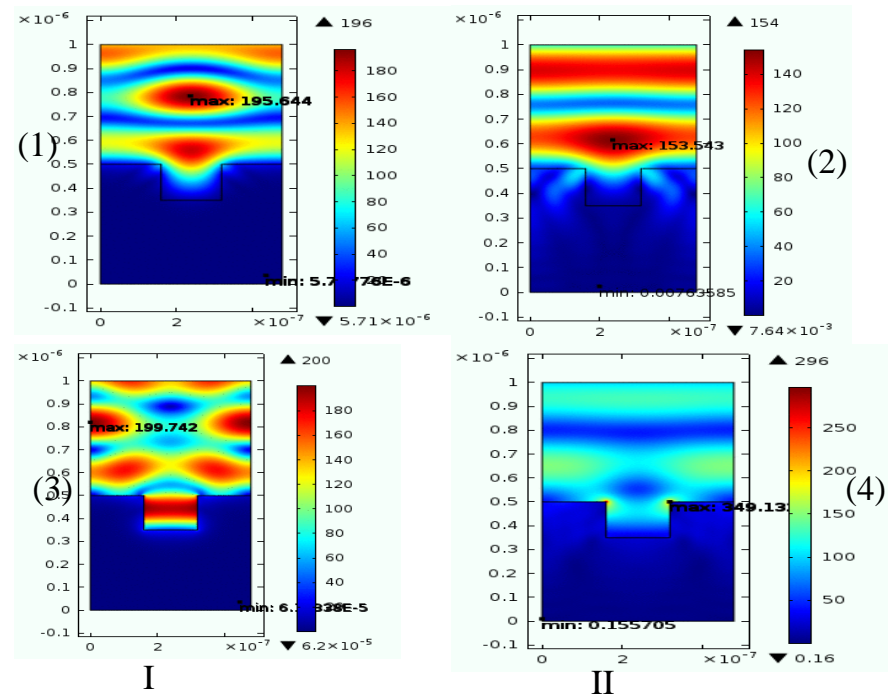

Fig. 5 - Electric field intensities, calculated for the filling factor $(f f=33.33 \%)$ : (1-2) for TE wave, (3-4) for TM wave. The profiles in column I and II are corresponding to the wavelength of $400 \mathrm{~nm}$ and $550 \mathrm{~nm}$ respectively

Table 2 - Max and Min Electric field calculated for the following filling factor (ff)

\begin{tabular}{|c|c|c|c|c|c|}
\hline \multicolumn{2}{|c|}{} & \multicolumn{2}{c|}{ TE wave } & \multicolumn{2}{c|}{ TM wave } \\
\hline \multirow{2}{*}{ filling factor $(f f)$} & wavelength & $\begin{array}{c}\text { Max Electric } \\
\text { field (V/m) }\end{array}$ & $\begin{array}{c}\text { Min Electric field } \\
(\mathrm{V} / \mathrm{m})\end{array}$ & $\begin{array}{c}\text { Max Electric } \\
\text { field (V/m) }\end{array}$ & $\begin{array}{c}\text { Min Electric } \\
\text { field (V/m) }\end{array}$ \\
\hline \multirow{2}{*}{$\begin{array}{c}\text { Unpatterned } \\
\text { layer }\end{array}$} & $\lambda=400 \mathrm{~nm}$ & 148.932 & $1.297 \mathrm{E}-6$ & 149.139 & $1.188 \mathrm{E}-5$ \\
\cline { 2 - 6 } & $\lambda=550 \mathrm{~nm}$ & 43.843 & 1.531 & 142.982 & 1.522 \\
\cline { 2 - 6 }$f f=33.33 \%$ & $\lambda=650 \mathrm{~nm}$ & 141.743 & 12.877 & 142.098 & 12.824 \\
\cline { 2 - 6 } & $\lambda=400 \mathrm{~nm}$ & $\lambda=400 \mathrm{~nm}$ & 195.644 & 199.742 & $6.133 \mathrm{E}-5$ \\
\cline { 2 - 6 } & $\lambda=550 \mathrm{~nm}$ & $\lambda=550 \mathrm{~nm}$ & 153.543 & 349.132 & 0.155 \\
\hline \multirow{3}{*}{$f=650 \mathrm{~nm}$} & $\lambda=650 \mathrm{~nm}$ & 139.493 & 445.171 & 1.521 \\
\hline
\end{tabular}

\subsection{Influence of the Period (a)}

To discuss the influence of the period, the absorption with various periods were calculated, and the results are shown in Fig. 6, where the depth and the filling factor are fixed at $150 \mathrm{~nm}$ and $50 \%$.

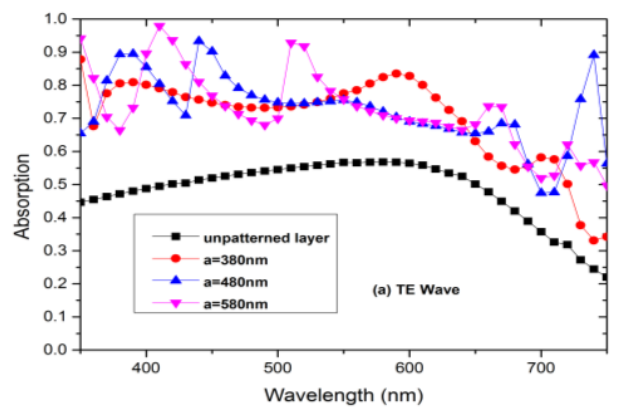

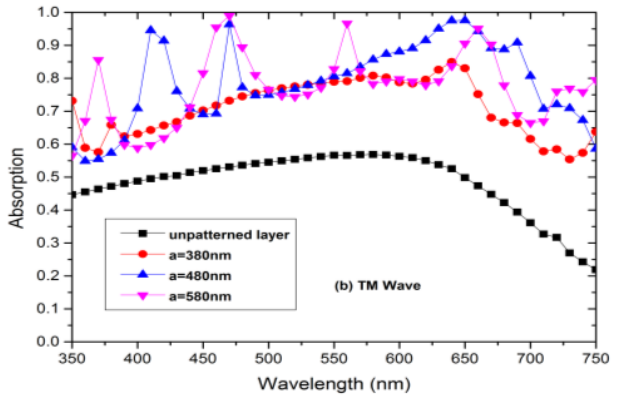

Fig. 6-Absorption for various periods, $d=150 \mathrm{~nm}$ and $f f=50 \%$ for (a) TE (b) TM wave

For wavelengths below $550 \mathrm{~nm}$ a slight improvement in absorption is observed when the period is increased and shows that there is very little change in 
absorption. Fig. 6 for TE and TM shows that the absorption peak at wavelengths at around $350-500 \mathrm{~nm}$ shifts towards the right with increase in period. The various periods do not significantly affect the absorption at wavelengths at around $540-650 \mathrm{~nm}$, but they have a notable effect at higher wavelengths.

In Fig. 7 we show the electric field inside the (a) TE wave (1-2) and (3-4) TM wave, under normally incident illumination. Remarkably, there is more energy penetrating into the absorption material.
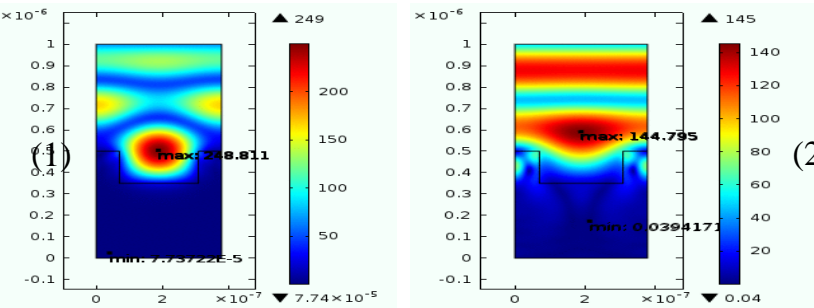
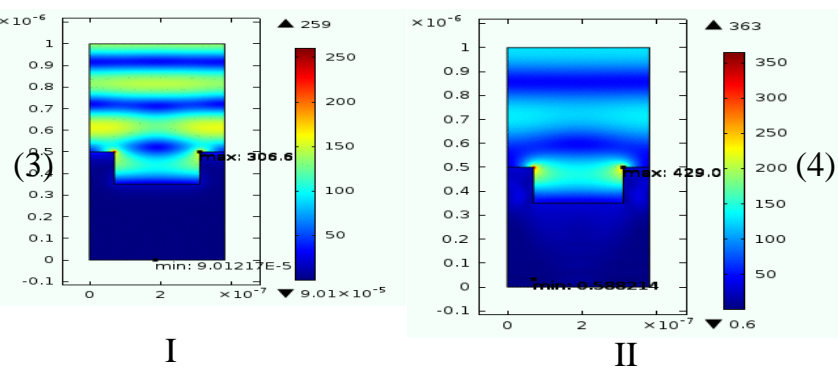

Fig. 7 - Electric field intensities, calculated for the period $(a=380 \mathrm{~nm})$ : (1-2) for TE wave, (3-4) for TM wave. The profiles in column I and II are corresponding to the wavelength of $400 \mathrm{~nm}$ and $550 \mathrm{~nm}$ respectively

Table 3 - Max and Min Electric field calculated for the following period (a)

\begin{tabular}{|c|c|c|c|c|c|}
\hline \multicolumn{2}{|c|}{} & \multicolumn{2}{c|}{ TE wave } & \multicolumn{2}{c|}{ TM wave } \\
\hline \multirow{2}{*}{ period $(\alpha)$} & wavelength & $\begin{array}{c}\text { Max Electric } \\
\text { field }(\mathrm{V} / \mathrm{m})\end{array}$ & $\begin{array}{c}\text { Min Electric field } \\
(\mathrm{V} / \mathrm{m})\end{array}$ & $\begin{array}{c}\text { Max Electric } \\
\text { field }(\mathrm{V} / \mathrm{m})\end{array}$ & $\begin{array}{c}\text { Min Electric field } \\
(\mathrm{V} / \mathrm{m})\end{array}$ \\
\hline \multirow{3}{*}{$\begin{array}{c}\text { Unpatterned } \\
\text { layer }\end{array}$} & $\lambda=400 \mathrm{~nm}$ & 148.932 & $1.297 \mathrm{E}-6$ & 149.139 & $1.188 \mathrm{E}-5$ \\
\cline { 2 - 6 } & $\lambda=550 \mathrm{~nm}$ & 43.843 & 1.531 & 142.982 & 1.522 \\
\cline { 2 - 6 } & $\lambda=650 \mathrm{~nm}$ & 141.743 & 12.877 & 142.098 & 12.824 \\
\hline \multirow{3}{*}{\begin{tabular}{c}
$\lambda=380 \mathrm{~nm}$ \\
\cline { 2 - 6 }
\end{tabular}} & $\lambda=400 \mathrm{~nm}$ & 248.811 & $7.737 \mathrm{E}-5$ & 306.062 & $9.012 \mathrm{E}-5$ \\
\cline { 2 - 6 } & $\lambda=650 \mathrm{~nm}$ & 144.795 & 0.039 & 429.073 & 0.588 \\
\hline
\end{tabular}

\subsection{Influence of the Incidence Angle ( $\theta)$}

The influence of incidence angle on absorption was calculated when the filling factor, period and depth were fixed at $50 \%, 480 \mathrm{~nm}$ and $150 \mathrm{~nm}$, respectively. The results are shown in Fig. $8 \mathrm{a}$ for TE and in Fig. 8b for TM wave.
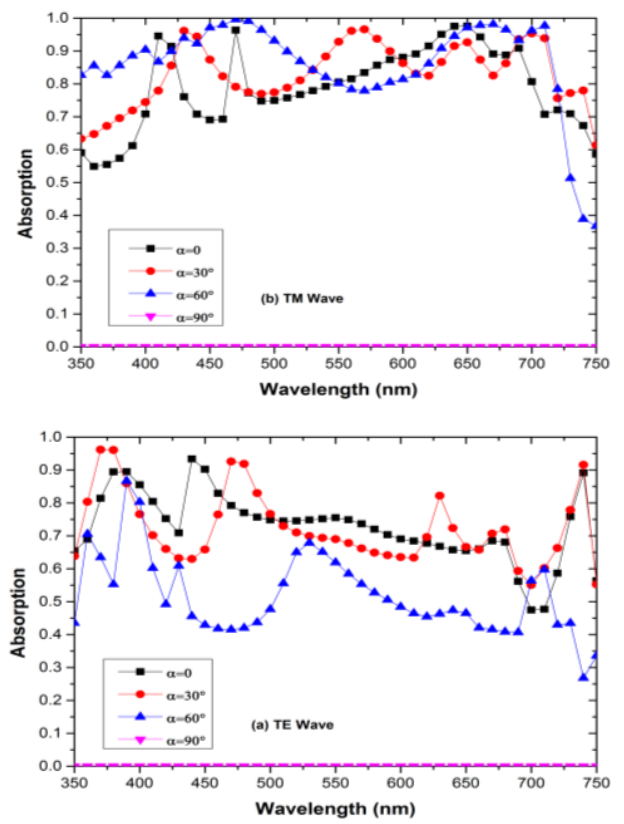

Fig. 8-Absorption with different incidence angles, $a=480 \mathrm{~nm}, d=150 \mathrm{~nm}$ and $f f=50 \%$ for (a) TE (b) TM wave tle the optical properties of the studied structure. It is seen that the absorption slightly increases for $\theta$ under $60^{\circ}$. For TE wave when incidence angle increases the absorption peak becomes very low.

It i evident from Fig. 9 that the high energy radiation is absorbed near the surface and fails to penetrate enough into the CP.
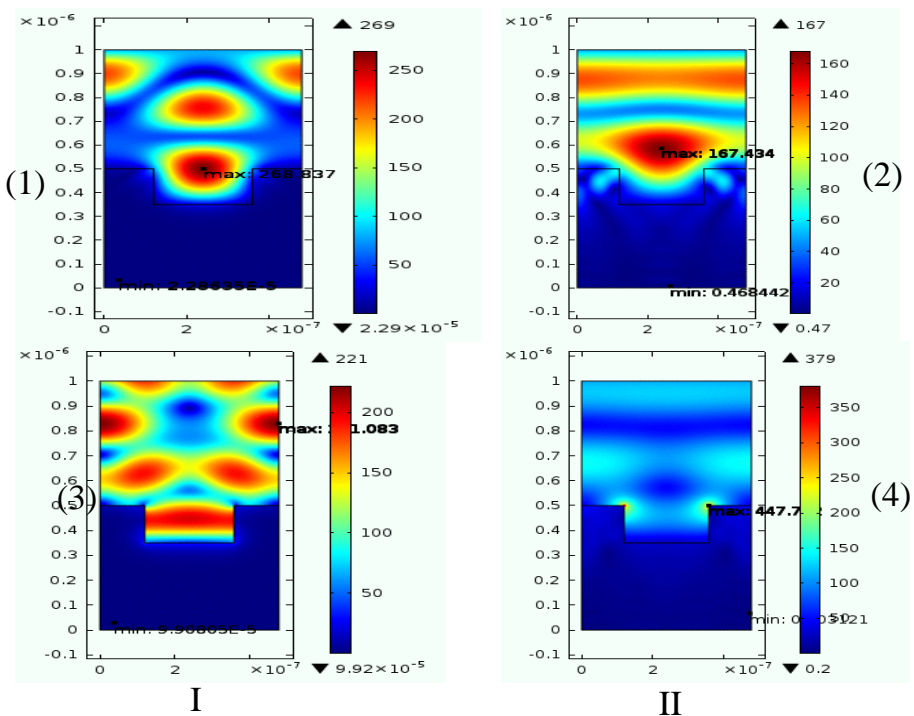

Fig. 9 - Electric field intensities, calculated for the incidence angle $\left(\theta=0^{\circ}\right)$ : (1-2) for TE wave, (3-4) for TM wave. The profiles in column I and II are corresponding to the wavelength of $400 \mathrm{~nm}$ and $550 \mathrm{~nm}$ respectively

Increasing the incidence angle changes only very lit- 
Table 4 - Max and Min Electric field calculated for the following incidence angle $(\theta)$

\begin{tabular}{|c|c|c|c|c|c|}
\hline \multicolumn{2}{|c|}{} & \multicolumn{2}{|c|}{ TE wave } & \multicolumn{2}{c|}{ TM wave } \\
\hline $\begin{array}{c}\text { Plane of } \\
\text { Incidence } \\
(\theta)\end{array}$ & wavelength & $\begin{array}{c}\text { Max Electric } \\
\text { field (V/m) }\end{array}$ & $\begin{array}{c}\text { Min Electric field } \\
(\mathrm{V} / \mathrm{m})\end{array}$ & $\begin{array}{c}\text { Max Electric field } \\
(\mathrm{V} / \mathrm{m})\end{array}$ & $\begin{array}{c}\text { Min Electric field } \\
(\mathrm{V} / \mathrm{m})\end{array}$ \\
\hline \multirow{3}{*}{$\theta=0^{\circ}$} & $\lambda=400 \mathrm{~nm}$ & 268.837 & $2.286 \mathrm{E}-5$ & 149.139 & $1.188 \mathrm{E}-5$ \\
\cline { 2 - 6 } & $\lambda=550 \mathrm{~nm}$ & 167.434 & 0.468 & 142.982 & 1.522 \\
\cline { 2 - 6 } & $\lambda=650 \mathrm{~nm}$ & 151.286 & 0.168 & 142.098 & 2.824 \\
\cline { 2 - 6 }$\theta=30^{\circ}$ & $\lambda=400 \mathrm{~nm}$ & 233.665 & 0.000 & 251.858 & $3.367 \mathrm{E}-5$ \\
\cline { 2 - 6 } & $\lambda=550 \mathrm{~nm}$ & 164.822 & 0.000 & 440.965 & 0.364 \\
\hline
\end{tabular}

\section{CONCLUSION}

In the present work we have investigated potential for light trapping by the use of one-dimensional photonic crystal structures. The aim object has been to identify structures that can provide good light trapping, that are to be used in amorphous silicon solar cell applications.

The influence of layers geometric parameters on the absorption is studied by using the Finite Element Method (FEM). The effect of the incident angle on the absorption also was discussed. The optimum parameters of the proposed structure are, period $a=480 \mathrm{~nm}$, a

\section{REFERENCES}

1. H. Ganesha

Shanbhogue,

C.L. Nagendra, M.N. Annapurna, S. Ajith Kumar, G.K.M. Thutupalli, Appl. Opt. 36(25), 6339 (1997).

2. Martin F. Schubert, Frank W. Mont, Sameer Chhajed, David J. Poxson, Jong Kyu Kim, E. Fred Schubert, Opt. Express 16 (08), 5290 (2008).

3. Lesley Chan, Dongseok Kang, Sung-Min Lee, Weigu Li, Hajirah Hunter, Jongseung Yoon, Appl. Phys. Lett. 104, 223905 (2014)

4. Huihui Yue, Rui Jia, Chen Chen, Wuchang Ding, Yanlong Meng, Deqi Wu, Dawei Wu, Wei Chen, Xinyu Liu, Zhi Jin, Wenwu Wang, Tianchun Ye, J. Vac. Sci. Technol, 08 (01), 46 (2011).

5. Jiun-Yeu Chen, J. Eng. Technol. Educat. 23 (03), 031208 (2011).

6. Harry A. Atwater, Albert Polman, Nat. Mater. 9 (3), 205 (2010).

7. Yuanyuan Li, Jian Pan, Peng Zhan, Shining Zhu, Naiben Ming, Zhenlin Wang, Wenda Han, Xunya Jiang, Jian Zi, Opt. Express 18(04), 3546 (2010).

8. Peter N. Saeta, Vivian E. Ferry, Domenico Pacifici, Jeremy N. Munday, Harry A. Atwater, Opt. Express 17(23), 20975 (2009). filling factor $f f=50 \%$ and depth $d=150 \mathrm{~nm}$. The absorption increases in the lower region where wavelengths around $480 \mathrm{~nm}$, is explained by the reduction of the effective index resulting from the structure. For wavelengths around $480-600 \mathrm{~nm}$, absorption is directly related to existing Fabry-Perot modes within the absorbent layer. In the region where the wavelength is greater than $600 \mathrm{~nm}$, it appears the additional absorption peaks weakly absorbing normally from the coupling of the incident light with the Bloch modes.

9. S.H. Zaidi, J. Gee, D.S. Ruby, IEEE Photovolt. Spec. Conf. 395 (2000).

10. J.G. Mutitu, S. Shi, C. Chen, T. Creazzo, A. Barnett, C. Honsberg, D.W. Prather, Opt. Express 16(19), 15238 (2008).

11. O. Painter, R.K. Lee, A. Scherer, A. Yariv, J.D. O'Brien, P.D. Dapkus, I. Kim, Science 284, 1819 (1999).

12. Leo T. Varghese, Yi Xuan, Ben Niu, Li Fan, Peter Bermel, Mi Nghao Qi, Adv. Opt. Mater. 1(10), 692 (2013).

13. Eli Yablonovitch, Phys. Rev. Lett. 58(20), 2059 (1987).

14. L. Zeng, P. Bermel, Y. Yi, B.A. Alamariu, K.A. Broderick, J. Liu, C. Hong, X. Duan, J. Joannopoulos, L.C. Kimerling, Appl. Phys. Lett. 93(22), 221105 (2008).

15. Benjamin Michael Curtin, Photonic crystal back-reflectors for light management and enhanced absorption in a-Si: $\mathrm{H}$ solar cells (Master of Science: Iowa State University: 2009).

16. A. Bielawny, C. Rockstuhl, F. Lederer, R.B. Wehrspohn, Opt. Express 17(10), 8439 (2009).

17. S. Guenneau, A. Nicolet, F. Zolla, S. Lasquellec, IEEE T. Magn. 38 (2), 1261 (2002). 